\section{ASPECTS OF THE DETERMINATION OF THE STRENGTH OF MATERIALS}

A

MEETING of the British Rheologists' Club was held on June 16 at the Royal Aircraft Establish. ment, Farnborough, Hants, in the Assembly Hall, through the courtesy of the director, Mr. W. S. Farren, the subject chosen being the "Strength of Materials". Dr. W. Douglas, head of the Materials Department of the Establishment, took the chair in the absence in the U.S.S.R. of the president, Prof. E. N. da C. Andrade. For those unfamiliar with the Establishment, it may be added that it is a directorate under the Controller of Research and Development of the Ministry of Aircraft Production. It is the chief research and experimental station under the Ministry, and it is concerned in all those fields of fundamental and applied science which influence the design, construction and performance of aircraft, military and civil, and in the equipment of aircraft.

Dr. M. C. Pryor, in a paper entitled "Wood and Glue", spoke during the morning session on the molecular and micro structure of timbers used in aircraft construction, and of the micro methods employed

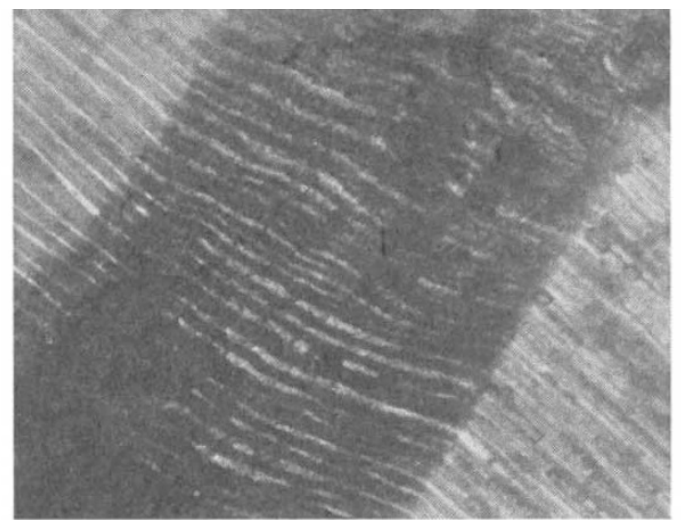

Fig. 1. Tension failude of Sitka sprdee, from an aIrcraft SPAR; LONGITUDINAL RADIAI, SECTION, SHOWING PLANES OF
FRACTURE. $\times 1,000$.

after an accident to establish the cause of mechanical failure. The movement of micelle aggregates during deformation is accompanied by rupture with the formation of fresh surfaces between the lignin and cellulose portions in the strained and damaged areas, and these fresh surfaces can be selectively stained. Failures by compression or tension are easily distinguishable microscopically, as originally shown by W. Robinson (Phil. Trans., B, 210, 49 ; 1920). Under compression, the tracheids in the zone of failure are considerably buckled. The zones of failure, which usually are well defined, run in planes at an angle of about $45^{\circ}$ to the compression stress. Under tension, failure develops along slip planes in the sub. stance of the cell walls, and these local shear failures occur in isolated points where the tube wall is weak, so that the failures are scattered up and down the members over quite a wide distance, perhaps for several inches. Some of the microphotographs shown at the meeting illustrating the general behaviour are reproduced herewith.

Mr. C. Gurney read a paper on the "Effect of Duration of Loading on the Strength of Brittle Materials". Some brittle materials, particularly mineral glasses, are known to be subject to static

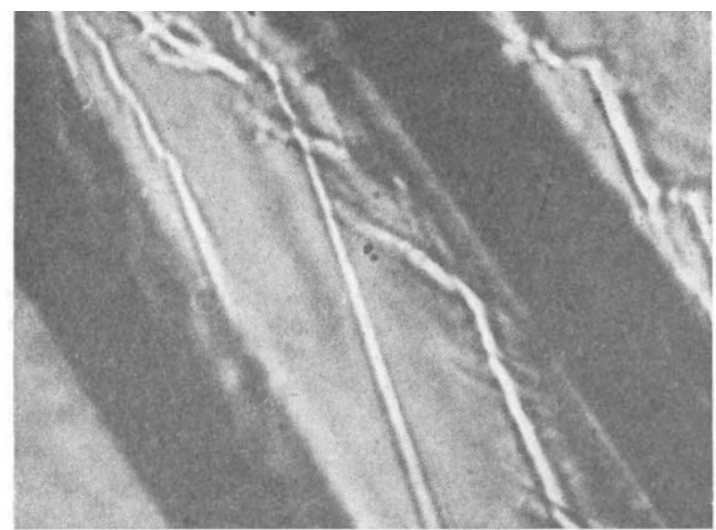

Fig. 2. ZONE OF FAIIURE IN DOUGLAS FIR, STAINED WITH ZINC CHLOR IODIDE. $\times 60$.

fatigue, the breaking load decreasing with duration of loading; for example, experiments on an annealed soda-lime glass showed that the strength decreased in the ratio $3: 1$ when the duration of loading was increased from $10^{-2}$ to $10^{4}$ sec. It is generally accepted that the weakness of brittle materials is due to cracks, as suggested by Griffith. A likely explanation of the time dependence of strength might be the gradual spread of cracks; Griffith, after considering the energy conditions necessary for the spread of cracks, concluded that crack-spreading could not occur until a critical load was reached; at this load the crack spread catastrophically, and failure was immediate. In these circumstances, the only previous explanation of failure after prolonged loading which is consistent with general principles is damage to the surface material by atmospheric attack. If glass is rapidly broken, there may be no time for atmospheric attack of the growing surfaces of the crack, so that a relatively high strength corresponding to the uncontaminated surfaces is obtained. A number of processes by which cracks may spread, consistent with the laws of thermodynamics, were described, and a new estimate of the strength of flawless materials was given. Stress reduces the height of atomic energy barriers, and immediate failure of a uniformly stressed material will occur when the average thermal motion is just sufficient to overcome the average onergy barrier. This condition is approximately that at which the latent heat of isothermal evaporation is zero.

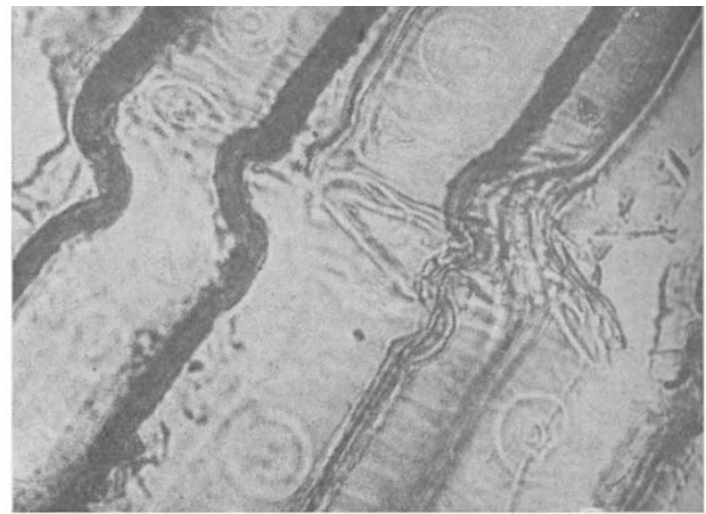

Fig. 3. COMPRESSTON FAILURE IN DOUGLAS FIR, SHOWING EARLY STAGE OF BCCKLING OF TRACHEIDS. $\times 400$ 


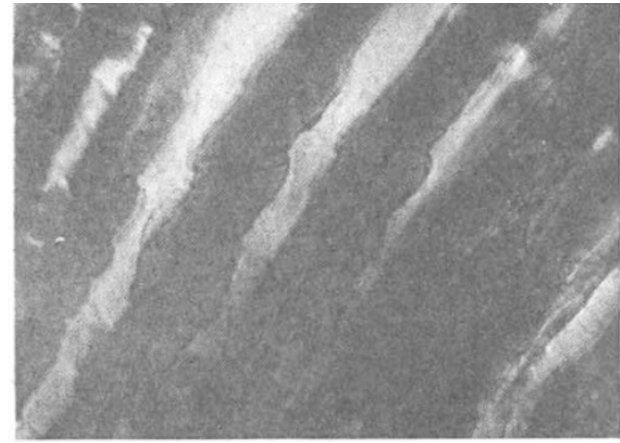

Fig. 4. COMPRESSION FAILURE IN DOUGLAS FIR, SHOWING SIP PLANES WITHIN THE CELI. WALIS. $\times 650$.

At lower stresses, failure is still possible, but it takes time. The stress-free vapour pressure of strong solids is extremely small, but it has been shown that the logarithm of the vapour pressure is approximately proportional to the square of the stress, so that the vapour pressure becomes appreciable at high stresses such as can occur at the end of cracks. The rate at which cracks spread by evaporation of the material has been estimated and found to be insufficient to account for the whole of the time effect with mineral glasses, tested under atmospheric conditions. In a material the atomic arrangement of which is in thermal equilibrium, increase in crack-length by splitting without atomic migrations can only occur at loads in excess of that given by the Griffith criterion. It has hitherto been thought that failure was immediate at the Griffith load, but it has been shown that, for materials having atomic constitution, the Griffith load is the lowest at which the erack may start to spread by splitting, the rate of spread being a function of the stress difference between that at the end of the crack and the maximum the material can withstand. For materials not in thermal equilibrium, the application of stress by reducing the height of energy barriers enables the approach to equilibrium to continue; and if this involves heterogeneous volume changes, internal stresses are set up which cause cracks to spread. Atmospheric attack, involving weakening of the surface material and possible solution of material at the ends of cracks-solubility increases very rapidly with stress--is likely to be an important factor in causing fracture under prolonged loading, when tests are made under ordinary atmospheric conditions.

In the discussion which followed, Dr. E. Orowan spoke on the mechanism of crack spreading and emphasized the importance of atmospheric attack. Dr. Maunder Foster mentioned the high stresses produced during grinding and their effect on reduction of molecular weight.

During the afternoon session, D1. B. Chalmers, in a paper with Mr. E. R. W. Jones, on the "Application of Statist ical Methods to Mochanical Test Results", gave a brief account of reasons for adopting a statistical approach to the interpretation of mechanical test results; and examples were given of significance testing, assessment of correlation and curve-fitting in relation to experimental results.

Strength tests are in general destructive, so that it is impossible to apply them to the component to be used. When they are non-destructive, the measurement is not of the property under consideration but of another property in some way related to it. In either case one requires a basis for inferring mechanical properties of the component from the known properties of the specimen tested. It was pointed out that the three reasons for doing mechanical tests are: (a) to provide design data; $(b)$ to determine whether two or more batches of material are significantly the same in the mechanical property examined ; and $(c)$ to provide the basis for establishing the rheological laws for the material.

It was emphasized that, in all statements of the numerical value of a quantity, it is desirable to quote both the mean and an estimate of the scatter, based on the standard deviation.

The importance of using impersonal methods for fitting curves was demonstrated by an example in which significantly different results were obtained by various observers interpolating on the same experimental points. In conclusion, a method of presenting results of fatigue tests was shown giving the stresses below which only a stated percentage of specimens would be expected to fail. The view was expressed that this is the logical method of expressing such information in cases where the data are to be used quantitatively for design purposes.

In a written contribution, Mr. R. L. Brown, of the Coal Utilisation Research Association, continued the discussion by referring to the statistical approach and the application of the Griffith crack theory to the strength of coal. In compression tests on cubical cylinders where the load is increased slowly until failure occurs, the worst crack determines the strength. Applying Frenkel and Kontorova's work on the 'worst' crack theory to the effect of size of specimen, it is found that data for coal are well represented (Fig. 5a).

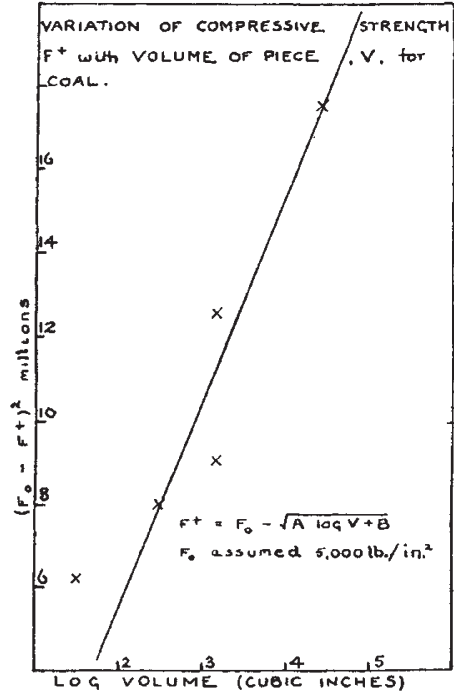

(a)

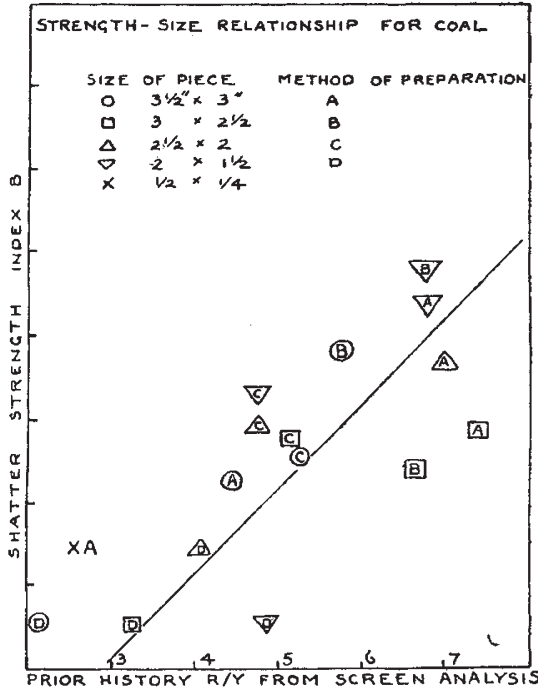

(b)
For size $x$ in., $R=$ per cent greater than size $(x)$ and $Y=$ yield of size $x$, per cent per inch, in consignment from which samples for test are taken.

Fig. 5. 
In shatter tests, strength can be assessed by the amount of breakage when a sample of a hundred or so pieces of coal is dropped $6 \mathrm{ft}$. on to an iron plate. Here the extent of cracks rather than the worst crack determines the strength. If it be assumed that the deformation caused by a sudden blow is elastic and that failure occurs when the strain energy exceeds a certain average value $E$ (the value of $E$ depends on the extent of pre-cracking and a hypothetical strain energy), then a formula can be derived for the shatter strength in terms of two factors ; first, the amount of breakage, and secondly the size distribution of the dust produced.

The size of the specimen now affects its strength in a rather different way. In Fig. $5 b$ the linear relationship between shatter strength and the size distribution of the consignment from which the coal for test is taken ( $R=$ per cent oversize, $Y=$ yield in per cent per inch) indicates that the shatter strength is proportional to the difference of the size subjected to test from the biggest material present (which is approximately $R / Y$ ) the departure being in the sense that the strength increases with decreasing size. This linear relation has been derived theoretically and will be published shortly.

The linear relationship shown in Fig. $5 b$ is interesting in that it enables the strength of the coal to be predicted from its previous history, so far as this is shown by the size distribution of the consignment. In other words, the handling of coal results in invisible breakage (or cracks and loss of strength) and visible breakage, and these two forms of breakage are balanced as shown.

\section{A CONSTITUTIONAL SYNTHESIS OF CHONDROSAMINE}

\author{
By DRS. SYBIL P. JAMES, F. SMITH, M. STACEY \\ and L. F. WIGGINS \\ Chemistry Department, University of Birmingham
}

$\mathrm{T}$

HE naturally occurring $d$-aminohexoses, glucosamine and chondrosamine, have long been known and are widely distributed in biologically important polysaccharides ${ }^{1}$. Any precise configuration of these substances, however, eluded chemists until 1939², when a derivative of glucosamine was synthesized by a method which proved conclusively that this aminosugar was configurationally related to glucose, a conclusion confirmed independently by X-ray crystallographic methods ${ }^{3}$. The configuration of chondrosamine or its derivatives, however, has not been elucidated hitherto. It is generally believed that chondrosamine, the amino-sugar of cartilage ${ }^{4}$, is related to $d$-galactose, with its amino-group on the second carbon atom ${ }^{5}$, and it can certainly have only one of two configurations, namely, that of $d$-galactose or $d$-talose, since the amino-sugar can be transformed with loss of its amino-group into galactose phenylosazon $e^{6,7}$.

The problem of deciding the configuration of chondrosamine has engaged our attention for some time, and by application of the technique of anhydroring formation and scission ${ }^{2}$, we are now able to report a synthesis of chondrosamine by a procedure which determines its constitution.

The method is dependent upon the discovery ${ }^{8}$ that anhydro-rings of the ethylene oxide type formed by the alkyl-oxygen fission of suitable esters ( $p$-toluene sulphonates) undergo ring-scission in both the possible directions, that is, by rupture of the bonds on either side of the oxygen atom of the threemembered ring, and in each case Walden inversion occurs when an entering anion attaches itself to the carbonium cation in the manner shown below.

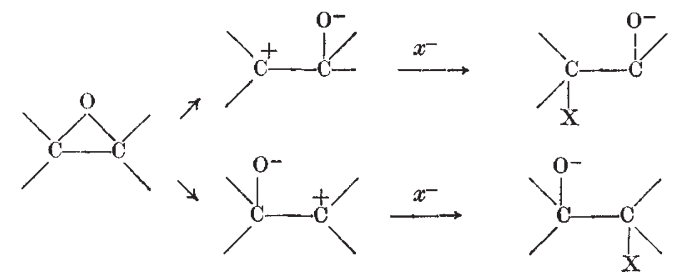

Thus the ring-opening of a derivative of $2: 3$-anhydroallose with sodium methoxide gives rise to 2-methyl altrose and 3-methyl glucose, and if the ring opening is effected through the agency of ammonia, then the corresponding amino-sugars are obtained. Applying this reaction to a derivative of $2: 3$-anhydro mannose, Haworth, Lake and Peat obtained a 3-amino-altrose (epiglucosamine) derivative and a 2-amino-glucose derivative, the latter being identical with that obtained from natural glucosamine. It was clear, therefore, that with a derivative of $2: 3$-anhydrotalose, ring fission with ammonia should lead to the formation of 3-amino-idose and 2-amino-galactose, which could then be compared with the corresponding derivatives of chondrosamine. Such a derivative of talose, 2 : 3-anhydro- $\beta$-methyltaloside (I), was made by one of $\mathrm{us}^{9}$ and subjected to ring-fission with ammonia. One compound only, namely, 3-amino$\beta$-methylidoside (II), could be isolated in crystalline form, presumably owing to the proportion of the second compound, 2 -amino- $\beta$-methylgalactoside (III), being small.

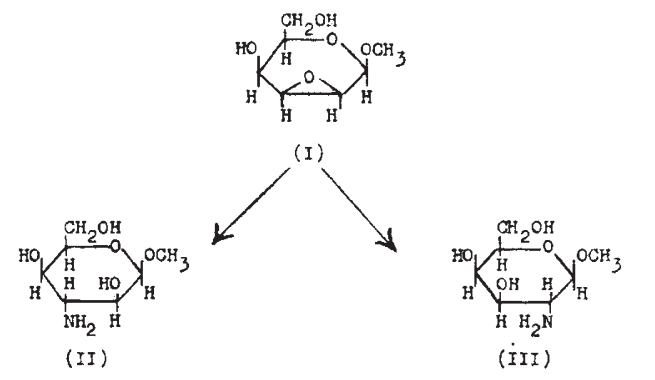

However, $1: 6-2: 3$-dianhydro- $\beta$-talose (IV) (cf. ref. 10), a compound obtained by the alkaline hydrolysis of 2-mesyl-1:6-anhydro- $\beta$-galactose, appeared to provide more suitable initial material. Its two anhydro-rings have widely different stabilities, the ethylene oxide ring being easily broken by both acid and alkaline reagents, whereas the $1: 6$-anhydrolinkage shows considerable stability towards alkaline reagents but can be broken by acid reagents. Therefore, by treatment of this dianhydride with ammonia, we expected to obtain $1: 6$-anhydro-2-amino- $\beta$ galactose (V) and I : 6-anhydro-3-amino- $\beta$-idose (VI), and by analogy with the investigations on $2: 3$ anhydro- $\beta$-methyltaloside, (VI) would be expected to be predominant. There would then remain to be settled the question as to which of the compounds was the idose derivative. This problem could be approached from a consideration of the products obtained by scission of $1: 6-3: 4$-dianhydro- $\beta$-talose ${ }^{11}$ 